\title{
Intertextualidad explícita en textos académicos de estudiantes universitarios. Un estudio exploratorio
}

\author{
Guillermo Soto*
}

\section{Resumen}

Las citas y otros dispositivos intertextuales cumplen diversas funciones en los textos científicos. En este estudio exploratorio, se analizan mecanismos intertextuales en escritos de estudiantes de segundo año de universidad tanto en ciencias naturales como sociales. Los resultados muestran que los sujetos emplean los dispositivos de modo reducido, tanto cuantitativa como cualitativamente. Se sugiere que el análisis de citas puede ser una herramienta de evaluación del desarrollo del discurso académico en la educación superior.

Palabras clave: discurso académico, alfabetización académica, intertextualidad.

\section{Abstract \\ Cites and other intertextual devices play several functions in scientific texts. In this exploratory study, I analyze intertextual devices in texts written by second year university students of natural sciences and social sciences. The results show that they use those devices restrictively, in quantitative and qualitative terms. I suggest that cite analysis can be used as a tool for evaluating the development of academic discourse in higher education.}

Key words: academic discourse, academic literacy, intertextuality. 


\section{Introducción}

La relación con otros discursos constituye un rasgo característico del lenguaje en uso (cf. Voloshinov, 1930/1992; Bajtin, 1986; de Beaugrande y Dressler, 1981; Calsamiglia y Tusón, 2007). Normalmente, los textos se insertan en redes - por lo general complejas y dinámicas- en que funcionan a la vez como respuestas parciales de otros textos y como incitación de futuros mensajes (cf. Voloshinov, 1930/1992), contribuyendo, de esta manera, tanto al desarrollo local de los procesos comunicativos como a la constitución, en un nivel mayor, de comunidades discursivas (Swales, 1990). En esta perspectiva, los textos pueden entenderse, metafóricamente, como nodos de una red comunicativa que está en constante gestación tanto local como globalmente. Más allá de la metáfora, estos nodos son la concreción de una actividad lingüística orientada a metas que es llevada a cabo por sujetos que, situados en contextos específicos, deben resolver determinados problemas.

Los discursos ajenos llegan a penetrar los mismos nodos textuales que, ya de manera latente ya de forma expresa, los incorporan en su tejido semántico lingüístico. Si bien en ocasiones estas voces ajenas ${ }^{1}$ se explicitan mediante recursos gramaticalizados como los procedimientos de discurso directo e indirecto (Maldonado, 1999), existe un continuo de procesos intertextuales ${ }^{2}$ con diverso grado de convencionalización y explicitud. En este sentido, la presencia de otras voces va desde las evocaciones generadas en el lector a partir de su interpretación del contenido semántico-pragmático del texto -sean éstas intencionadas o no por el autor- hasta los recursos gramaticales que marcan explícita-

1 Entenderemos por "voz ajena" toda instancia de discurso proferida originalmente por alguien distinto del autor, por el propio autor en otro momento o por el propio autor asumiendo que es otro. La voz ajena podrá mantener las características de la enunciación original o limitarse al enunciado o a la proposición proferidos (cf. Voloshinov 1930/1992, Maldonado 1999).

2 Se emplearán indistintamente los términos "procesos" y "recursos". 
mente la presencia de otra voz. Entre ambos extremos, podemos ubicar procesos intertextuales que, a nuestro entender, son característicos de ciertos géneros discursivos; esto es, recursos convencionalmente establecidos para indicar la referencia a otras voces en ciertas variedades de discurso. Estos procesos, sin estar gramaticalizados, pueden describirse como esquemas o patrones de textualización genéricamente situados, cuyo manejo es esperable por parte de (o exigible a) los miembros de la comunidad discursiva (Soto, 2005).

El presente trabajo indaga en los procedimientos empleados en textos de estudiantes universitarios de carreras de ciencias naturales y sociales para incorporar explícitamente otras voces en su texto, un aspecto de la intertextualidad manifiesta (Hyland, 2002). La investigación descansa sobre el supuesto de que un rasgo característico de la prosa científica, tal y como ésta se manifiesta textualmente, consiste en la existencia de patrones explícitos y complejos de incorporación de las voces ajenas en el texto propio. Mediante el empleo de estos recursos - que pueden variar entre los distintos campos disciplinarios-, el investigador intenta ubicar sus proposiciones en la red de proposiciones ya establecidas (en mayor o menor grado) en el campo. En otras palabras, estos procesos explicitan las relaciones que establece el investigador entre sus proposiciones y otras proposiciones del campo disciplinario ${ }^{3}$.

En términos muy generales, y sin intentar agotar las funciones del discurso especializado, puede proponerse que, a través del texto científico, el investigador pretende, normalmente, alterar, en algún aspecto, el campo de conocimientos existente en un dominio disciplinario o interdisciplinario específico. Esto supone, primero, una evaluación y toma de partido con respecto al subconjunto de conocimientos pertinente, $y$, segundo, una incorporación de las proposiciones textuales informativas ${ }^{4}$ en el campo de conocimientos de la disciplina ${ }^{5}$. Los recursos en estudio participan en estas tareas toda vez que no sólo explicitan la incorporación de una proposición ajena sino que también permiten expresar la posición que el autor tiene con respecto a las proposiciones ajenas (i. e. su evaluación o valoración).

En consonancia con lo expuesto, pensamos que el manejo adecuado de los procesos en estudio supone no sólo conocimiento de las convenciones formales del género en cuestión (procedimientos de cita, referencias bibliográficas, etc.). Junto a ello, es un índice del conocimiento que

3 La idea de que las citas son expresión de la intertextualidad en textos académicos se encuentra también en Myers (1999) y Hyland (2002). Para Myers, estos "vínculos intertextuales" establecen un campo de interacción.

4 Es decir, de aquellas proposiciones nuevas con respecto al campo disciplinario.

5 La misma idea es expuesta en Leydesdorff y Amsterdamska (1990) 
el autor tiene del subconjunto de supuestos pertinentes en su campo de investigación. De ser esto efectivo, el estudio del desarrollo de los procesos empleados para incorporar explícitamente otros discursos en el texto académico contribuiría -indirectamente, por cierto- a la comprensión y evaluación del grado de incremento del conocimiento disciplinar por parte de los sujetos.

Un estudio de este tipo encuentra antecedentes en las investigaciones en retórica y composición, que han contribuido a entender la composición en las disciplinas como una tarea sociocognitiva compleja que se relaciona íntimamente con el desarrollo disciplinar de los estudiantes (cf. Nelson, 1992; Berkenkotter, Huckin y Ackerman, 1989).

\section{La intertextualidad y el discurso académico: el caso de las citas}

En una concepción social y cognitiva de los géneros discursivos, la intertextualidad desempeña un papel crítico. En efecto, lejos de constituir un componente marginal de la competencia comunicativo-textual de los hablantes, la intertextualidad y el dialogismo son procesos fundamentales de la construcción textual, entendida como una tarea sociocognitiva en que el sujeto construye un discurso y una identidad discursiva que responden y apelan a otros discursos e identidades. Como afirma Hyland (2002: 41)

[L]os escritores típicamente se posicionan a sí mismos y a sus ideas en relación con otras ideas y textos en sus comunidades, y esto los ayuda a legitimar su inclusión (membership) y establecer sus identidades individuales a través del discurso ${ }^{6}$.

El empleo de las citas, entendidas como la referencia "a otra palabra, frase, oración, párrafo o título de texto desde la perspectiva del artículo que cita" (Leydesdrof, 1998, § 2)7 es una expresión abierta de intertextualidad y constituye, a la vez, uno de los rasgos característicos del discurso académico (cf. Myers, 1999; Leydesdorff, 1998).

Como es de esperar, los manuales de redacción académica entregan información relativa a este proceso, destacando su función como recurso

$6 \quad$ Las traducciones son responsabilidad del autor de este trabajo.

7 Emplearemos la expresión "cita" para abarcar tanto las citas propiamente tales como las paráfrasis. En el mismo sentido, cf. Thompson (2001). Para Leydesdrof (1998), "las citaciones se refieren a otra palabra, frase, oración, párrafo o título de texto desde la perspectiva del artículo que cita" (§ 2). 
que permite distinguir entre los planteamientos propios y los ajenos. Así, por ejemplo, en el manual de escritura académica del "Writing center" de la Universidad de Wisconsin-Madison (2003), la entrada "Citing references in your paper" indica que "la mayor parte de la escritura académica requiere que el autor reúna, evalúe y use el trabajo de otros", otorgando el "crédito debido" a los otros autores. El alto valor que se asigna al manejo adecuado de las citas se desprende de las sanciones que acompañan su mal uso. Tras presentar la función atribuida a la cita, el manual referido agrega que el no cumplimiento de la obligación de reconocimiento constituye plagio, advertencia recurrente en textos similares ${ }^{8}$.

Con frecuencia, los manuales, junto con indicar la función atribuida a las citas y otros recursos de documentación y referencia, proceden a entregar información formal relativa al sistema de citas y referencias (cf., por ejemplo, AIT Extensión; Serafín, 1994; Nowodworsky y Sarmiento, s.f., entre otros ${ }^{9}$ ). Aun cuando en ocasiones esta caracterización formal alcanza cierta sofisticación -como en el caso de Thompson (2001) ${ }^{10}$-- el problema merece una atención más detenida y funcional tanto en lo concerniente al texto científico propiamente tal como en lo relativo a los textos académicos escritos por estudiantes ${ }^{11}$.

8 El manual del Language Center de la AIT de Tailandia define en los siguientes términos plagio: "Esto [plagio] significa usar las palabras, ideas o información de otros sin hacer referencia a ellos -en otras palabras, presentarlos como propios. Cualquier trabajo que no esté referido se asume que es suyo, por tanto asegúrese de que esto es verdad. El plagio es una falta grave y puede dar lugar a castigos, incluyendo la expulsión del Instituto. Para evitarlo, emplee citas y paráfrasis con las referencias adecuadas. Cuando lea, tome notas cuidadosas de sus fuentes, incluyendo toda la información bibliográfica que necesite para escribir una referencia completa de las fuentes". Como se advierte, la ausencia de referencias esperables se considera plena prueba del plagio cometido por un autor, y el plagio, una infracción grave contra la ética académica. Con todo, es posible que existan diferencias culturales en esta materia, como a veces se ha señalado. Contrástese el fragmento anterior con el siguiente, de Serafini (1994): "Cuando en el texto propio se citan literalmente las palabras de otro sin entrecomillarlas, se comete un plagio. Aunque en general no se descubren, los plagios son desaconsejables: rara vez se incurre en ellos por malicia; más a menudo su origen se encuentra en la ingenuidad y en el desorden de los propios apuntes, en los que se habían consignado las palabras exactas de un texto sin entrecomillarlas, en lugar de un resumen o una paráfrasis de las mismas" (p. 339).

9 Existen distintos estilos. En el mundo anglosajón destacan el de la APA (American Psychological Association), el del CBE (Council of Biology Editors) y el de la MLA (Modern Language Association); todas éstas, convenciones de extenso empleo en el ámbito internacional. A éstas hay que añadir las existentes, aunque de manera, al parecer, más difusa, en el mundo hispanoamericano, y las propias variantes de diversas publicaciones. Para una revisión sumaria, y crítica, del tratamiento de las citas en textos anglosajones sobre inglés con propósitos académicos, véase Thompson (2001).

10 A partir de la dicotomía de Swales (1990) entre citas integrales y no integrales, Thompson propone una subcategorización para cada tipo. Así, las no integrales se dividirían en de fuente, de identificación, de referencia y de origen. Las integrales, por su parte, en de verbo controlador, de nominalización, y de no citación.

11 En parte, esta atención al problema de las citas tiene que ver con su papel en los procesos de clasificación académica. Los índices de citación desempeñan un papel 
En efecto, en cuanto a los textos científicos, se ha destacado la pluralidad de funciones desempeñadas por las citas (cf. Leydesdorff y Amsterdamska, 1990; Swales, 1990; Teufel y Monees, 2000). En primer término, como indican Teufel y Moens (2000), en el texto científico las aseveraciones se distinguen según éstas se atribuyan a otros investigadores, al campo en general (trasfondo) o al propio autor, y el sistema de citas desempeña un papel crucial en esta determinación. Como suele ocurrir en el discurso científico, el problema fundamental consiste, primero, en saber determinar qué se explicita y qué no (en este caso, qué es trasfondo y qué se atribuye a un autor), y segundo, en identificar con claridad las proposiciones auténticamente informativas que el autor avanza en el texto.

La investigación muestra que hay, sin embargo, más en las citas que esta función atribucional. Las citas desempeñan funciones de corte social e incluso institucional, cumplen funciones retóricas, apoyan argumentose instancian fórmulas superficiales. Ya Serafini (1994), por ejemplo, un texto claramente didáctico que no se aboca al discurso científico, identifica las funciones de "comentar con precisión un texto" (338), argumentar a partir del prestigio de un autor; "exhibir la propia cultura"; evitar la paráfrasis, y demostrar el conocimiento de los textos.

Cozzens (apud Leydesdorff y Amsterdamska, 1990) propone que en los artículos científicos las citas poseen una naturaleza doble. De una parte, establecen vínculos entre textos y operan, en consecuencia, en el sistema retórico o cognitivo de la ciencia ya que lo relevante, en estos casos, es el contenido cognitivo de la relación ${ }^{12}$. Por otro lado, establecen relaciones entre autores y funcionan en el sistema de recompensas o el sistema social de relaciones entre científicos. De acuerdo con Leydesdorff y Amsterdamska (1990) esta dicotomía es dinámica y admite lo que denominaremos recategorizaciones ${ }^{13}$.

En lo que dice relación con su función cognitivo-retórica, Teufel y Moens (2000) proponen una subcategorización de funciones de las citas a partir de su tricotomía inicial. Como ya hemos dicho, la distinción básica en estos autores se da entre proposiciones (oraciones) atribuidas al propio trabajo, al trasfondo compartido de la disciplina y al trabajo de otros. Las oraciones que se atribuyen al autor pueden o no describir el propósito

importante en la vida académica y científica, sobre todo en disciplinas altamente estandarizadas, como la biología, y en los centros de mayor prestigio. Ha surgido, así, la denominada "citacionología" o "análisis de citaciones", subdisciplina de la cienciología. Con respecto a la relevancia para la enseñanza de los géneros académicos, véase Thompson (2001).

12 Leydesdorff y Amsterdamska (1990) prefieren hablar de sistema cognitivo en vez de sistema retórico.

13 En el sentido de las recategorizaciones en gramática. 
central del trabajo. Si lo hacen, son citaciones de propósito (aim); si no lo hacen, pueden o no tratar de la estructura externa del artículo. Las que lo hacen son "textuales" y las que no lo hacen, "propias". Estas últimas contienen la información comunicada por el escritor. Con respecto a las atribuciones al trabajo de otro, estas serán "de contraste" si describen un aspecto negativo del otro trabajo, o un contraste o una comparación con el propio trabajo (esta función también se especifica en Swales, 1990). En caso contrario, servirán de antecedente intelectual ("base") si sirven para fundar o apoyar el propio trabajo. Finalmente, las que no caigan en esta última categoría (esto es, las que provean una descripción neutra del otro trabajo) caerán en la categoría "otro".

Como ya se ha indicado, las citas desempeñan un rango mucho más amplio de funciones, algunas de ellas de tipo social más que cognitivo. Las citas de contraste, de acuerdo con Swales (1990), contribuyen al establecimiento de la propia investigación en un nicho determinado: al destacar las carencias, errores, ausencias, presentes en los otros trabajos, el agente aclara un campo que, luego, puede pasar a ocupar. En este sentido, la cita puede emplearse tácticamente para ayudar en la resolución de un problema comunicativo específico que encara el autor: instaurar un espacio que pueda ocupar con su investigación. Con frecuencia, las citas son también una señal de la competencia del autor en el campo (piénsese, por ejemplo, en las citas cuando se reseña un dominio específico). Ciertamente, la caracterización provista hasta aquí es incompleta; sin embargo nos entrega cierta base para el estudio de las citas en estudiantes universitarios.

\section{Método}

Con el fin de identificar el empleo cuantitativo y cualitativo de las citas en estudiantes universitarios, se procedió a analizar, en primer lugar, 14 informes correspondientes a estudiantes de un curso de Ecología de $2^{\circ}$ año de la carrera de Ingeniería en Recursos Naturales Renovables de la Facultad de Ciencias Agronómicas de la Universidad de Chile (2002). Los informes se presentaban, visualmente, con un formato semejante al de un artículo de estructura IMRD (Introducción, Método, Resultados y Discusión) o semejante. Temáticamente, trataban estudios de distintos ecosistemas a partir de visitas en terreno. También se analizaron 5 informes del curso de Equilibrio de Mercado, correspondientes a estudiantes de $2^{\circ}$ año de la carrera de Economía de la Universidad de Chile (2001). En este caso, se trató de informes analíticos en que se discutían conceptos de las ciencias sociales y que se asemejaban más bien a ensayos. Aunque el número de textos de economía es menor que el de ecología, se trata de textos más extensos en que se esperaría una mayor presencia 
de recursos de intertextualidad. La opción por estudiantes de segundo año obedeció a que estos se encuentran en las fases preliminares de su formación en un campo disciplinario. No se trabajó con estudiantes de primer año pues se consideró que estos estaban recién saliendo de la educación secundaria y comenzaban su exposición a la enseñanza universitaria. Los autores de los escritos son estudiantes de la universidad más tradicional del país y la que tiene los índices más altos en investigación y publicaciones; en ese sentido, puede plantearse que pertenecen a la elite académica de su generación. Otro criterio de opción tuvo que ver con el hecho de que mientras un grupo estaba formado por estudiantes de ciencias naturales, el otro lo conformaban estudiantes de ciencias sociales. Lamentablemente, por el escaso número de informes que se pudo conseguir, no pudo realizarse una selección interna de los escritos. Tampoco se tuvo información sobre el rendimiento de los estudiantes, aunque en el caso de economía, las notas asignadas por el profesor del ramo aportaban alguna información.

El análisis se realizó manualmente y se empleó como criterio de reconocimiento de la cita, la atribución de un mensaje (cita, paráfrasis, concepto) a un otro específico ("Pérez ha propuesto...") o genérico ("muchos creen que..."). Además de las citaciones, se analizó la cantidad de publicaciones referidas en la sección Referencias (o equivalente). El objetivo de este análisis fue enriquecer la caracterización del nivel intertextual explícito de los informes y establecer una comparación con las citas. La referencia de las publicaciones empleadas constituye también un rasgo de los textos académicos (y en consecuencia de los científicos). Posteriormente, los datos se agruparon por trabajo y por sección. Después de la exposición de los resultados, se analizó la cantidad de citaciones por informe y el número de publicaciones en la sección Referencia (o equivalente), estableciendo comparaciones e intentando reconocer patrones.

\section{Resultados}

\subsection{Informes de Ecología}

En lo que sigue, se analizan los resultados relativos a citas y publicaciones referidas, encontrados en 14 informes del curso de Ecología, correspondientes a estudiantes de $2^{\circ}$ año de la carrera de Ingeniería en Recursos Naturales Renovables de la Universidad de Chile (2002). Los datos se ordenan por trabajo (numerados EC1, EC2, etc.). La Tabla 1 presenta la frecuencia de citaciones por texto. 
Tabla 1. Citaciones por texto en estudiantes de ciencias naturales

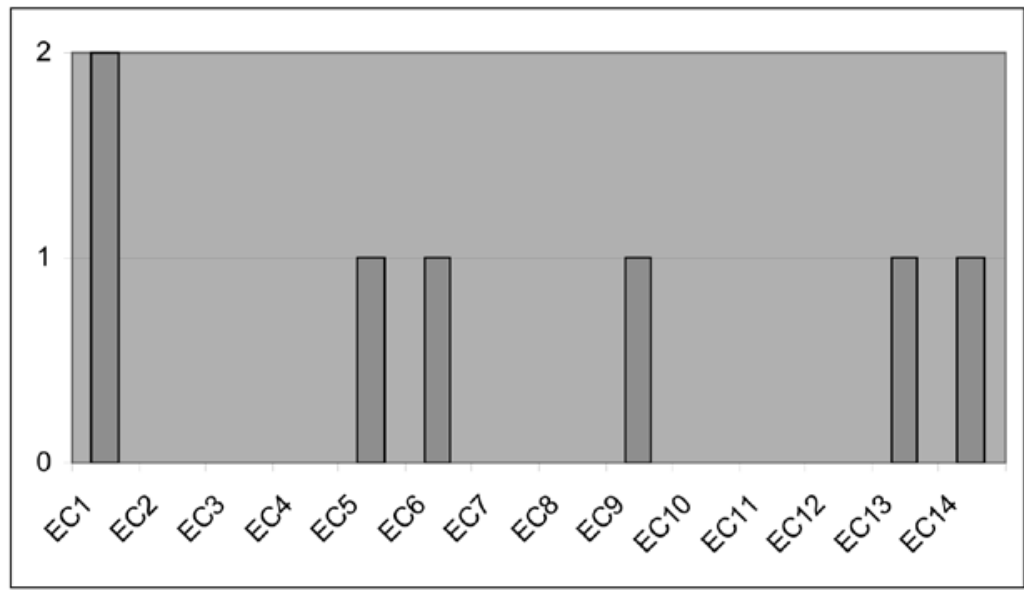

Como se advierte en la Tabla, hay escaso empleo de citaciones. Sólo en 6 de los 14 informes se cita explícitamente a otros autores (42.8\%). En dos casos (EC9 y EC13) no se trata realmente de citas sino de atribuciones de autoría de clasificaciones, como se observa en (1):

(1) Este tipo de vegetación es característico de la zona central y pertenece a la Región del matorral del bosque esclerófilo (clasificación de Rodolfo Gajardo) [EC9, sección Discusión].

Si no consideramos estas instancias, los informes con citaciones se reducen a sólo 4 (28,6\%).

Los recursos empleados para dar cuenta explícitamente de mensajes ajenos son variados. En el primer caso de EC1, la cita va entre comillas y se atribuye al saber general del campo disciplinar, sin atribución específica. Como es usual en español, se emplea para tal efecto la tercera persona plural, que indica la indeterminación del sujeto: "han sido definidos como":

(2) Los ecosistemas son unidades en las cuales se estudian flujos de energía y materiales y han sido definidos como "El sistema total en sentido físico incluyendo no solamente el complejo de organismo, sino también el conjunto de factores físicos formando eso que denominamos el ambiente de bioma, los factores del hábitat en el sentido más amplio". [EC1, sección Introducción]

En el segundo caso de EC1, se trata de una cita entre comillas ${ }^{14}$. La atribución se realiza poniendo el nombre del autor entre paréntesis: "(Ga-

14 En estricto rigor, faltan las comillas al inicio de la cita. Esto se ha interpretado como un lapsus calami. 
jardo)". En el caso de EC5 y en el de EC6, la citación rescata el contenido pero no la forma del mensaje, por lo que no va entre comillas. La atribución se realiza ya poniendo el nombre del autor y el año de la publicación referida entre paréntesis, "(Vásquez, 1993)", ya poniendo solo el nombre del autor, "... Hoffman)". El ejemplo 3 muestra el procedimiento en EC5:

(3) En el nivel de organización, el ecosistema funciona como un sistema abierto (definido dentro del espacio y el tiempo) donde existe una integración y autoregulación entre los elementos no vivientes del ecosistema (factores físicos, químicos y fisicoquímicos) con los factores biológicos multiespecíficos, es decir con una diversidad de especies (Vázquez, 1993). [EC5, sección Introducción]

En la instancia de EC14, la cita va entre comillas y la atribución se realiza poniendo el nombre del autor y el año de la publicación referida entre paréntesis: "(Tansley 1935)".

En cuatro casos las citas se emplean para entregar definiciones o caracterizaciones conceptuales (en dos de estos casos se emplea el verbo "definir"). En un caso la cita se utiliza para entregar información de tipo descriptivo respecto de una especie vegetal. En dos instancias, finalmente, se nombra a autores de clasificaciones.

El listado de referencias bibliográficas, ilustrado en la Tabla 2, constituye otro mecanismo para explicitar la presencia de voces ajenas en el texto.

Tabla 2. Publicaciones incluidas en sección de referencias bibliográficas, textos de ciencias naturales

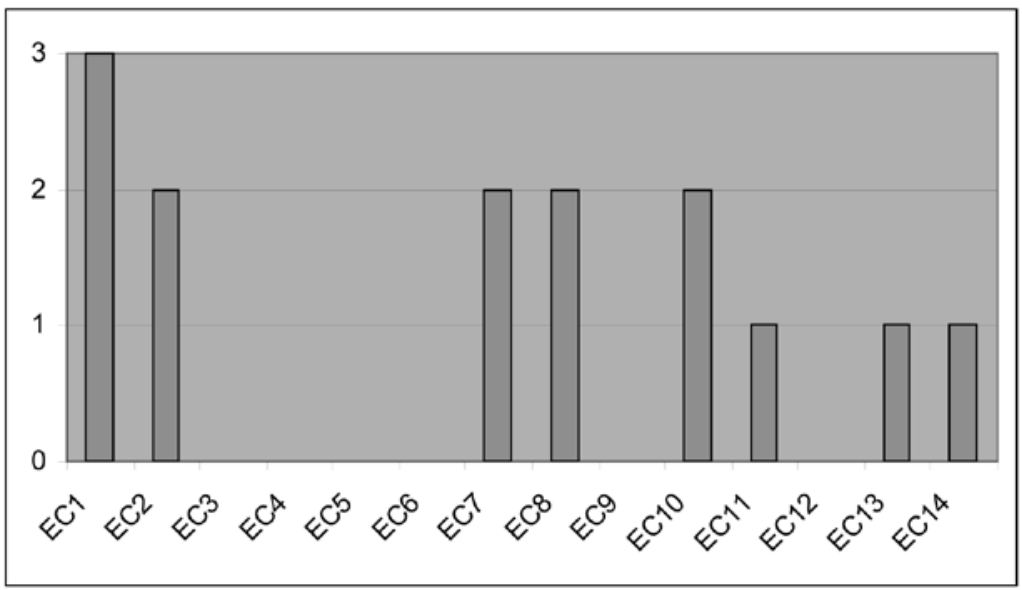


Como puede advertirse en la Tabla, en 8 de los 14 informes existe sección de Referencias $(57,1 \%)$. No siempre las publicaciones que figuran en Referencias son referidas en el cuerpo del trabajo (p. ej., EC2, EC7, EC8, EC10, EC11, donde no hay citaciones en el informe). Por otro lado, hay trabajos que refieren en el cuerpo a ciertos autores o a ciertas obras, sin que luego exista una sección de Referencias (EC5, EC6, EC9).

\subsection{Informes de Economía}

A continuación, se analizan los resultados correspondientes a citas y publicaciones referidas, recabados de 5 informes del curso de Equilibrio de Mercado, correspondientes a estudiantes de $2^{\circ}$ año de la carrera de Economía de la Universidad de Chile (2001). Los datos se agrupan por trabajo (numerados EQ1, EQ2, etc.). La Tabla 3 presenta la frecuencia de citaciones por texto.

Tabla 3: Citaciones por texto en estudiantes de ciencias sociales

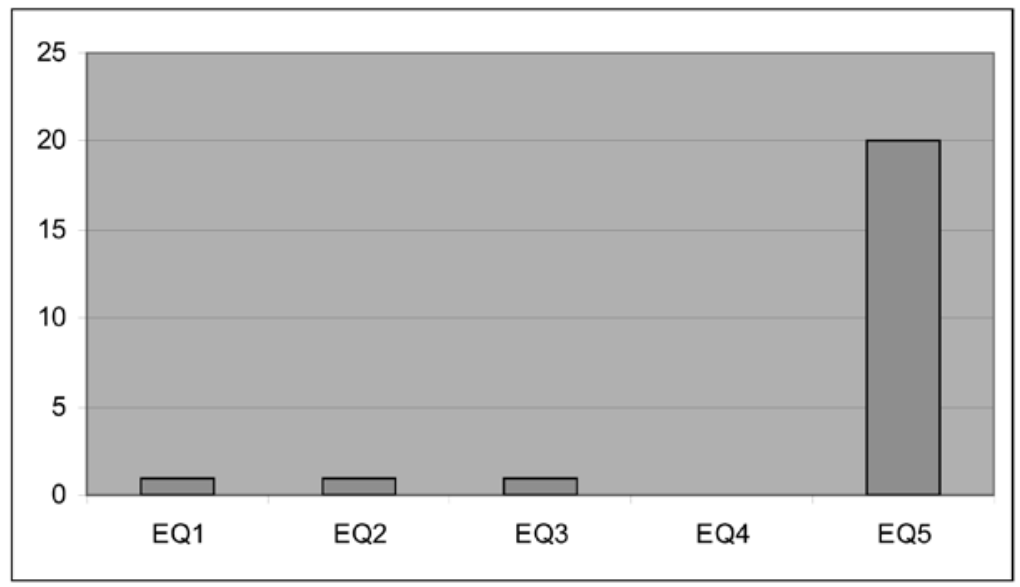

Como se observa en la Tabla, en general hay un uso muy escaso de citaciones, salvo en el texto EQ5, que por sí solo contiene más citaciones que todos los otros juntos. Un uso interesante en estos textos, se observa en EQ1, que cita una opinión de alcance muy general de un autor de prestigio:

(4) "Si no aprendemos de la historia podríamos vernos obligados a vivirla de nuevo. Pero si no planeamos el futuro, nos veremos obligados a soportarlo. Y eso podría ser mucho peor" (Alvin Toffler) [EQ1, sección Análisis final]

El procedimiento se repetirá en EQ5. Tanto EQ3 como EQ4 refieren a autores que especifican perspectivas, ya generales, que no requieren 
más que la mención de autores ampliamente conocidos, como en EQ 3, donde se describe una situación "siguiendo a Marx y Engels", ya específicas, como en EQ4, que emplea un recurso de mayor frecuencia en los textos especializados: "Según Cinthy B. Lloyd (1979)".

Con respecto a las citaciones de EQ5, estas aparecen en las distintas secciones del trabajo y desempeñando un amplio rango de funciones, no todas ellas características del discurso especializado. Además de aquellas que reiteran los patrones observados en los textos de ciencias naturales, el autor emplea con cierta frecuencia la cita bibliográfica a pie de página, aunque no siempre conforme a las normas usuales de citación. El siguiente ejemplo ilustra el procedimiento, frecuente, en efecto, en las ciencias sociales y las humanidades:

(5) Organismos Internacionales dicen que más de la cuarta parte de la población mundial vive en condiciones de extrema pobreza, o que las 225 personas más ricas del mundo tienen una riqueza combinada igual al ingreso anual del $47 \%$ más pobre $(2.500 \mathrm{mi}-$ llones de habitantes) ${ }^{1}$

a. [En cita de pie de página, n. 1, en (1)] Columna de Roberto Avila, La Nación, 09 de Junio [sic] del 2001. [EQ5, sección Pregunta e hipótesis a tratar]

En ocasiones, la cita indica solo a un autor reconocido, sin especificar la fuente, como en el siguiente ejemplo:

(6) El propio Norbert Wiener, inventor de la cibernética, al señalar las propiedades de esta nueva ciencia, pronosticó allá por 1948 sus consecuencias: la extinción del trabajo, las características del nuevo poder en base a la tecnología, las transformaciones de la inteligencia y los nuevos parámetros del espacio tiempos [sic]. [EQ5, sección Trabajo informal en un mundo globalizado]

Como ya se indicó, se cita también a autores de prestigio, no siempre relacionados con el tópico específico de investigación. Por otro lado, se hacen referencias a un saber general, que es objeto de evaluación: Dicen algunos. Estos usos serán comentados más adelante, en la sección de Discusión, donde se ilustrarán los casos. El texto también apela al lector para involucrarlo, en un empleo interactivo que, aunque no común en textos especializados, es consistente con la mayor conciencia intertextual que este manifiesta.

(7) Muchos se estarán haciendo esta misma pregunta, ya que para el sistema capitalista los marginados son un estorbo. [EQ5, sección ¿Qué hacer con los marginados?] 
En lo relativo a las referencias bibliográficas, como se puede observar en la Tabla 4, en 4 de los 5 informes existe sección de Referencias (80\%).

Tabla 4: Publicaciones incluidas en sección de referencias bibliográficas, textos de ciencias sociales

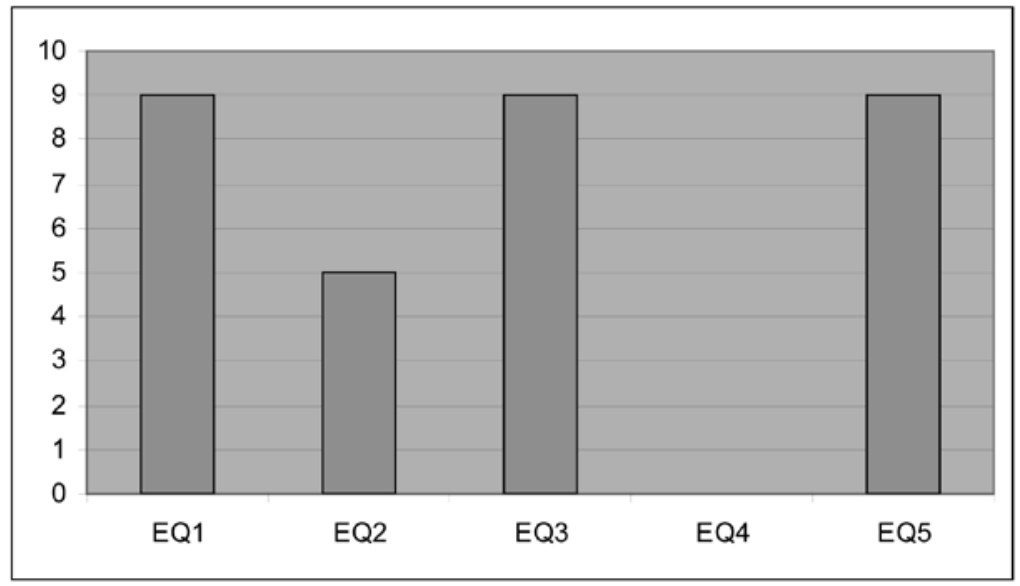

Como en el caso de los textos EC, no siempre las publicaciones que figuran en la sección Referencias son referidas en el cuerpo del trabajo (p. ej., EQ1, EQ2 y EQ3, donde la cantidad de referencias bibliográficas supera con creces las citaciones). Por otro lado, en EQ4, aunque hay una cita no existe sección de referencias. Finalmente, cabe destacar que en EQ5 gran parte de las referencias bibliográficas se entrega en notas de pie de página.

\section{Discusión}

En general, los resultados sugieren, primero, que hay poco empleo de citas y referencias a otros en los textos estudiados, y, segundo, que estas citas y referencias presentan poca variedad funcional. En otras palabras, existe una pobreza tanto en la frecuencia de citas como en la gama de funciones que estas desempeñan.

Resulta interesante observar que la fuerte restricción funcional de las citas se asemeja a patrones característicos de adquisición del lenguaje. En efecto, normalmente, los niños tienden a emplear los esquemas recién adquiridos con el fin de satisfacer ciertas funciones específicas. Es en el desarrollo ulterior cuando el niño va asignando progresivamente, con cada vez mayor flexibilidad, nuevas funciones a las viejas formas lingüísticas, pasando de un modelo una-forma/una-función a otro del 
tipo una-forma/muchas-funciones y una-función/muchas-formas (Ravid y Tolchinsky, 2002).

Más allá de las explicaciones debidas a los tipos de informes que debían preparar los sujetos, es posible que el escaso empleo obedezca a un conocimiento todavía escaso del dominio conceptual de la disciplina. Un indicador -que reconocemos débil- en este sentido es el hecho de que la información contenida en citas y paráfrasis es presentada, en la enorme mayoría de los casos, como información cierta. En otras palabras, es notoria la ausencia de recursos de modalización epistémica. En efecto, faltan en el corpus verbos y construcciones que disminuyan el grado de certeza del mensaje (del tipo de "sugieren", "parecen proponer que", etc.). Junto a lo anterior, son también escasos los mensajes ajenos que se presentan para ser criticados, un uso frecuente en textos científicos maduros (citas de contraste de Teufel y Moens 2000). El único escrito en que se da esta función -EQ5-atribuye las opiniones criticadas a agentes genéricos (p. ej., "muchos"), o a estereotipos, de manera irónica. En conclusión, los autores entregan, en la enorme mayoría de los casos, citas, paráfrasis y referencias que apoyan la línea argumental y expositiva del texto, y en el único caso en que se entregan mensajes ajenos que van en contra de esa línea, el autor utiliza un esquema más propio de textos políticos o abiertamente ideológicos que de textos de disciplinas científicas.

Otro rasgo destacable, específicamente en los casos EQ, es el empleo de citas, paráfrasis y referencias de carácter más bien general que no se relacionan con el tema específico de la investigación. En ocasiones, estas citas parecen estar al servicio de comentar y apoyar la idea principal del texto o incluso la postura ética del autor. Hablaremos, en este caso, de citas manifiestamente axiológicas como la siguiente, de EQ5:

(8) Alguna vez Gandhi nos recordó: "la tierra tiene bastante para las necesidades de todos, pero no para la avaricia de algunos". [EQ5, sección Conclusiones]

En casos como este, el autor establece una relación de apoyo entre la argumentación global del texto y el mensaje citado, que opera como argumento de autoridad y recurso estilístico. Con todo, la relación entre la cita y el mensaje o el propósito del texto es figurativa. Es interesante observar, en este punto, la recurrencia de la argumentación centrada en la calidad de la persona: "El mismo Kofi Annan, secretario general de la ONU, dijo en una entrevista ..." (EQ5), "El mismísimo Sebastián Piñera hace un llamado" (EQ5).

Si bien la información recabada en la presente investigación es preliminar, es sugerente relacionarla con rasgos de las publicaciones científi- 
cas del siglo XIX. Como señala Swales (1990), hacia fines del siglo XIX las citas, aunque no escasas, "raramente se relacionaban con los hallazgos o los tópicos específicos investigados por los autores" (114). A partir de 1910, la pertinencia restringida será un factor decisivo en la selección de las citas. Esto significará que, en un primer momento, disminuirá el número de citas. El cambio fundamental, en todo caso, radicará en que las citas estarán relacionadas directamente con el tópico específico del artículo.

Otra característica que recurre en las citas axiológicas encontradas en los escritos estudiantiles de economía es su empleo como marcadores de cierre textual. Se trata de una función que suele indicarse en manuales de estilo, libros de redacción y textos de lengua castellana y comunicación. Es posible que su uso en estos textos obedezca a la proyección de una táctica aprendida en la educación secundaria. El hecho de que se trate de textos de carácter más bien ensayístico posiblemente no hizo sino favorecer esta proyección.

Ciertamente, en la escritura de estos informes, los estudiantes no se enfrentaron a las situaciones ni, consecuentemente, a las exigencias de los expertos de las respectivas disciplinas. No obstante, las tareas presentan rasgos que parecen característicos de los dominios. En un caso, la información generada a partir de una visita de campo, y en el otro, la argumentación en torno a un tema económico-social específico. Por ello, al menos en parte, los estudiantes debieron abordar problemas semejantes a los de los expertos, aunque, obviamente, con otro nivel de complejidad y en otra situación de vida.

\section{Conclusiones}

A modo de síntesis, se exponen a continuación las conclusiones que pueden derivarse de este estudio preliminar. En relación con la cantidad de citas empleadas en los textos, esta es escasa, con excepción del escrito EQ5. Con respecto a la función de las citas, hay un fuerte predominio de aquellas que apoyan la exposición/argumentación del autor. En el único caso en que se añade la función de aportar citas que serán rebatidas (citas de contraste), el autor parece ajustarse a un esquema de discurso público político o manifiestamente ideológico. En economía, aparecen citas axiológicas que apoyan la postura ideológica del autor, apelando a un argumento de autoridad. Estas citas suelen desempeñar también una función estilística de cierre del texto, lo que se asocia a escritos periodísticos o con alguna pretensión literaria. En relación con la disciplina, las citas parecen indicarnos que los sujetos no han alcanzado aún un 
grado de conocimiento del campo disciplinario tal que les permita interactuar críticamente con él y formular sus propuestas sobre un trasfondo interpretado por ellos. Son frecuentes los errores formales y de estilo, especialmente en la sección de referencias bibliográficas.

El panorama expuesto apunta a que los sujetos se encuentran en las primeras fases del desarrollo de la competencia genérica. Por esta razón, no manejarían aún los procesos de cita y referencia que caracterizan al discurso científico, y tenderían, posiblemente, a simplificar este componente o a proyectar procesos que se utilizan en otros géneros discursivos que han empleado con anterioridad.

Si bien son necesarios estudios más amplios para validar la utilidad de este tipo de análisis, el ejercicio que aquí se expone sugiere que el estudio de las citaciones en textos de alumnos universitarios puede ser una herramienta sencilla y útil para aquilatar el grado de dominio del discurso especializado e, indirectamente, el nivel de incorporación en la comunidad discursiva específica, dos objetivos de la formación disciplinaria.

\section{Bibliografía}

AIT Extension.

Language Center. Referencing. http://www. languages.ait.ac.th/EL21REFP.HTM (consulta, 1 de septiembre de 2003).

Bajtin, M. (1986). "The problem of speech genres". Speech genres and other late essays. Austin, Estados Unidos: University of Texas Press, 60-102

Berkenkotter, C., Huckin, T. \& Ackerman J. (1989). Social context and socially constructed texts: the initiation of a graduate student into a writing research community. Technical Report No 33, National Writing Project. Web: http://www. writingproject.org/downloads/csw/TR33.pdf (consulta, 10 de julio de 2003)

De Beaugrande, R. \& Dressler, W. (1981). Introduction to text linguistics, Londres, Inglaterra: Longman.

Hyland, K. (2002). Teaching and researching writing. Londres, Inglaterra: Longman.

Leydesdorff, L. (1998). “Theories of Citation?" Scientometrics, 43(1), 5-25

Leydesdorff, L. \& Amsterdamska, O. (1990). "Dimensions of citation analysis". Science, Technology \& Human Values, 15, 305-335. 
Maldonado, C. (1999). "Discurso directo y discurso indirecto". En I. Bosque y V. Demonte (coords.), Gramática descriptiva de la lengua española. Madrid, España: Espasa-Calpe, 3549-3596.

Myers, G. (1999).

"Interaction in writing: Principles and problems". En C. Candlin y K. Hyland (eds.), Writing: Text, proceses, and practices. Londres: Longman, 40-61.

Nelson, J. (1992). Constructingaresearchpaper:Astudy ofstudents'goals and approaches. Technical Report № 59, National Writing Project. Web: http://www.writingproject. org/downloads/csw/TR59.pdf (consulta, 10 de julio de 2003)

Nowodworsky, E. y Sarmiento, P. s.f. El trabajo oral y escrito. Guía para estudiantes, empleados y profesionales, Santiago, Chile: Universitas Ediciones

Ravid, D. \& Tolchinsky, L. (2002). “Developing linguistic literacy: A comprehensive model". Journal of Child Language, 29(2), 417-447.

Serafini, Ma T. (1994). C Cómo se escribe. Barcelona: Paidós.

Soto, G. (2005). "Los géneros discursivos como tecnologías cognitivas". Rasal (Revista de la Sociedad Argentina de Lingüística), 1, 37-51.

Swales, J. (1990). Genre analisis: English in academic and research settings. Cambridge: Cambridge University Press.

Teufel, S. \& Moens, M. (2000). “What's your and what's mine: Determining intelectual attribution in scientific text". Joint SIGDAT Conference in Empirical Methods in Natural Language Processing and Very Large Corpora. 7-8 de octubre, Hong-Kong. Web: http://acl.eldoc.ub.rug. nl/mirror/W/WOO/WOO-1302.pdf (cons. 10 de julio de 2003).

Thompson, P. (2001). "Looking at citations: Using corpora in English for Academic Purposes". Language Learning \& Technology, 5(3), 91-105.

Thompson, P. \& Tribble, C. (2001). "Looking at citations: Using corpora in English for academic purposes". Language Learning \& Technology, 5 (3). 91-105

University of Wisconsin-Madison Writing Center. (2003). Handbook. Web: http:// www.wisc.edu/writing/Handbook/Documentation. html (consulta, 10 de julio de 2003).

Voloshinov, V. (1930/1992). El marxismo y la filosofía del lenguaje. Madrid: Alianza. 